REAL-TIME CONTROL OF SULPHUR DIOXIDE EMISSIONS FROM AN INDUSTRIAL AREA

P. Melli

IBM Scientific Center, Rome, Italy

P. Bolzern, G. Fronza, and A. Spirito

Centro Teoria Sistemi CNR - Istituto Elettrotecnica ed Elettronica, Politecnico, Milan, Italy

RR-81-34

December 1981

Reprinted from Atmospheric Environment, volume 15 (1981)

INTERNATIONAL INSTITUTE FOR APPLIED SYSTEMS ANALYSIS

Laxenburg, Austria 
Research Reports, which record research conducted at IIASA, are independently reviewed before publication. However, the views and opinions they express are not necessarily those of the Institute or the National Member Organizations that support it.

Reprinted with permission from Atmospheric Environment 15: 653-666 (1981). Copyright $\odot$ Pergamon Press Ltd.

All rights reserved. No part of this publication may be reproduced or transmitted in any form or by any means, electronic or mechanical, including photocopy, recording, or any information storage or retrieval system, without permission in writing from the copyright holder. 


\section{FOREWORD}

The International Institute for Applied Systems Analysis conducts research on a variety of problems relating to the environment, its concerns ranging from understanding its problems and the phenomena underlying them through building models of these phenomena to using these models in solving the problems.

This paper summarizes the recent work on the control of sulphur dioxide pollution stemming from emissions in the industrial area of Porto Marghera in the Venetian region.

Italian legislation on air pollution around industrial stacks presently prescribes a 30minute ambient standard for sulphur dioxide. Therefore, any emission control plan must be based on a relatively refined model of $\mathrm{SO}_{2}$ dispersion that accounts for the conspicuous short-term fluctuations of ambient pollutant concentrations.

This paper develops and tests a control scheme that prescribes intermittent emission reduction by using clean fuels when the dispersion model forecasts that the near-future ambient concentration levels will be too high. A similar control technique is widely used in Japan, and, in empirical form, is also used by the Italian energy authorities to govern emissions from their power plants.

While implementation of this technique may raise organizational and legal issues in some cases, cost-effectiveness analysis shows that it may be either a valid alternative or complement to permanent emission controls.

JANUSZ KINDLER

Chairman

Resources and Environment Area 


\title{
REAL-TIME CONTROL OF SULPHUR DIOXIDE EMISSIONS FROM AN INDUSTRIAL AREA*
}

\author{
P. Melli \\ IBM Scientific Center, Rome, Italy \\ and \\ P. Bolzern, G. Fronza and A. Spirito \\ Centro Teoria Sistemi CNR - Istituto Elettrotecnica ed Elettronica, Politecnico, Milano, Italy
}

(First received 2 May 1980)

\begin{abstract}
Real-time emission control is an air quality policy which is alternative to permanent emission reduction. In general terms, it consists of controlling emission only when a forthcoming episode is forecast. Thus, control costs are lower than costs due to permanent abatement. The natural application is a case characterized by a limited number of polluting sources. In more specific terms, a real-time emission control scheme consists of the following operations at the beginning of each time interval (hour, say).

(i) Collect current concentration and meteorological measures by a monitoring network.

(ii) Forecast future values of relevant local meteorological variables.

(iii) On the basis of information about current concentration values, forecast meteorology and scheduled emissions predict future concentrations.

(iv) If future concentrations exceed some reference level, reduce the scheduled emissions.

The paper describes a case study [application of scheme (i)-(iv)] to $\mathrm{SO}_{2}$ pollution from the industrial area in the Venetian lagoon region). The general characteristics are the following:

The meteorological predictors [step (ii)] are simple stochastic methematical predictors.

The concentration predictor [step (iii)] is based on a complex forecast algorithm (Kalman predictor). It is derived from the "stochastic version" of the numerical solution of the advection-diffusion partial differential equation.

The control policy [step (iv) ] is assumed to consist of mixing with cleaner fuel under the constraint of maintaining the production scheduled by each polluting plant.

The results of the case study are supplied as cost-effectiveness curves (cost versus effectiveness of the control action).
\end{abstract}

\section{INTRODUCTION}

A large number of mathematical models are now used in air quality management. Nearly all these models are concerned with long-run decision-making problems, such as allocation and design of polluting sources or assignment of permanent emission abatement to each polluter. In most cases, the "modelling approach" simply consists of applying a mathematical representation of pollutant dispersion (e.g. a Gaussian-type representation) to the different decision-making alternatives (i.e., to different locations and heights of the stacks and/or to different abatement policies). In a few cases, the search of a rational decision is more systematic, namely is made through a formal optimization model (e.g. Guldmann and Shefer, 1976; Emanuel et al., 1978; Seinfeld and Kyan, 1971; Atkinson and Lewis, 1974).

However, long-run decision-making is just one side of air quality management problems, and, in some situations it may even not be the most relevant aspect,

* Research supported by the International Institute of Applied Systems Analysis (IIASA), Laxenburg, Austria. in view of the following considerations.

(a) Many air pollution cases are due to already existing sources, namely there is, in general, no source allocation and design problem.

(b) Apart from technological difficulties, a pollution regulation policy, which is only based on the permanent abatement of emission may lead to relevant drawbacks either from the side of costs or from the side of effectiveness. In fact, if permanent emission reductions are established so that the most severe pollution episodes are acceptable the cost of abatement is likely to be extremely high. On the contrary, if permanent abatement is moderate, average pollution will be lowered but severe meteorological situations are still likely to bring pollutant concentrations well above tolerable standards.

An approach, which allows considerable reduction in the drawbacks mentioned in (b) consists of replacing permanent emission abatement by (or, at least, of combining it with) real-time emission control (see for instance Shepard, 1970; Leavitt et al., 1971). In general terms, real-time control is a sequential short-run management procedure, which, at the beginning of 
each time step (hour, say) is based on the following operations (see also Soeda and Omatu, 1979):

(i) By a monitoring network collect the present and recent values of pollutant concentrations and significant meteorological variables in the area under consideration.

(ii) On the basis of such information about the current local meteorological situation (and, possibly, on the basis of synoptic forecast, see for instance Barbieri et al., 1979) predict future values (for the next 2 or $4 \mathrm{~h}$, say) of the significant meteorological variables. In principle, this can be accomplished either automatically, i.e., by running mathematical predictors of the meteorological variables on a computer, or, simply, by experience.

(iii) On the basis of the information about pollutant concentrations (mentioned in (i)), forecast meteorology (mentioned in (ii)) and scheduled future emissions, predict future concentration levels. Again, this can be accomplished either automatically, through a mathematical concentration predictor (see for instance Bankoff and Hanzevack, 1975; Finzi et al., 1978; Finzi et al., 1979; Finzi et al., 1980; Bacci et al., 1981) or simply, by experience.

(iv) If the predicted concentrations (mentioned in (iii)) exceed preassigned levels, reduce the scheduled emissions in accordance with some rational abatement policy.

As clear from (i) - (iv), the philosophy of real-time control is to take an action on the emissions only in a situation of forthcoming "episode" (actually, of forecast forthcoming "episode"). Therefore, the control action turns out to be intermittent, thus yielding a conspicuous cost-saving with respect to permanent abatement.

Naturally an hour-by-hour control scheme like (i) (iv) can in practice be set up only where a limited number of sources are present (= industrial area). Moreover, the implementation of the scheme requires the establishment by authority both of reference pollution levels [the "preassigned levels" mentioned in (iv)] and of the "rational control policy" [also mentioned in (iv)].

The present paper describes a case study, concerning the real-time control of $\mathrm{SO}_{2}$ pollution from the industrial area of Porto Marghera, in the Venetian lagoon region. The details concerning procedure (i) (iv) (which monitoring network for step (i), which meteorological predictors for step (ii), which predictor of the concentration field for step (iii), which emission reduction policy for step (iv) ) are respectively specified in the following four sections. In particular, the most relevant general characteristics of the present real-time control scheme are the following:

The meteorological predictors mentioned in (ii) are (see Section 3) simple stochastic mathematical predictors, either probabilistic or ARMA (Auto Regressive Moving Average, see, e.g., Box and Jenkins, 1970).
The concentration predictor mentioned in (iii) is a complex stochastic predictor, since it is derived (see Section 4) from the "stochastic version" of a numerical solution scheme of the advection-diffusion partial differential equation (see also Bankoff and Hanzevack, 1975).

The control action mentioned in (iv) is assumed (see Section 5) to consist of emission reduction by fuel mixing or replacement under the constraint of maintaining the scheduled production for each polluting plant.

The results of the analysis are summarized in the last section, in terms of cost-effectiveness curves. Specifically, the preassigned concentration level mentioned in (iv) is used as a parameter. For each values of such parameter, the cost of real-time control (measured by the percent extra-expenditure due to the control action, namely to the introduction of low sulphur fuel) is reported versus the effectiveness of control (effectiveness defined by an overall index, which measures the decrease of pollution due to the control action).

\section{DESCRIPTION OF THE VENETIAN LAGOON POLLUTION PROBLEM AND MONITORING NETWORK}

The area under consideration (Fig. 1) is located in the north-eastern part of Italy, on the north coast of the Adriatic Sea. It includes part of the extreme end of the Padana Plain and part of the Venetian lagoon, precisely it consists of the urban centers of Mestre, Marghera and Venice and the industrial area of Porto Marghera (one of the largest in Europe). The urban centers of Mestre and Marghera, situated on the mainland, have developed very rapidly in the last three decades and have now a surface area of about $10 \mathrm{~km}^{2}$. The industrial area is about $20 \mathrm{~km}^{2}$ and its main activities include oil-refining, petrochemical production, metallurgical processing of iron and other metals, and electric power production. $6 \mathrm{~km}$ from the mainland, in the middle of the Lagoon, is the historical center of Venice, covering an area of $6 \mathrm{~km}^{2}$ and standing on a cluster of small islands.

The $\mathrm{SO}_{2}$ pollution problem in the region has already been analyzed in a number of studies. For instance the influence of meteorological factors on pollution levels has been investigated by Zannetti et al. (1977). Moreover, mathematical models, both deterministic (Runca et al., 1976; Marziano et al., 1979) and stochastic (Finzi et al., 1979) have been used for simulating or predicting long-term or short-term average $\mathrm{SO}_{2}$ concentrations in the area.

In particular, Zannetti et al. (1977) analyzed the occurrence of episodes both in the historical center of Venice and in the mainland. It turned out that most of the pollution episodes occur in the industrial area, while the few concerning Venice correspond to winter 


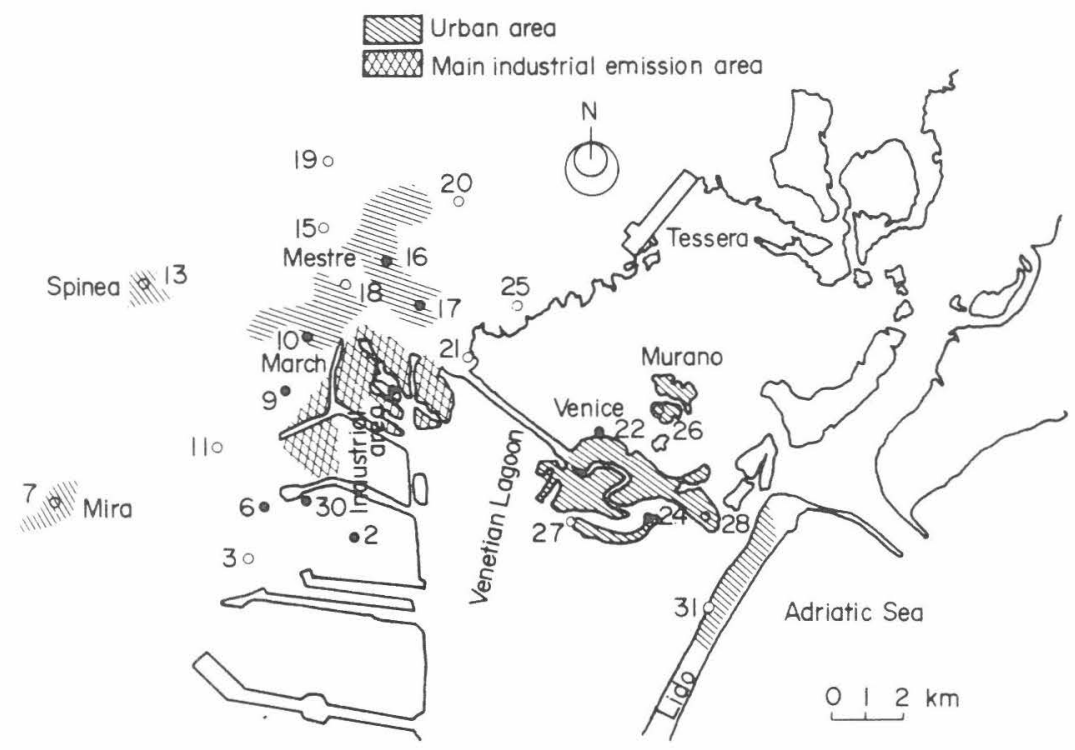

Fig. 1. The Venetian lagoon region.

winds blowing from the industrial area and have smaller intensity. Hence, using concentration measurements in the historical center would not add much information to a control scheme of air quality. Thus the present model has been implemented only in correspondence with the region shown in Fig. 2. Of course, this choice has significantly reduced the computational effort.

The available emission, meteorological and $\mathrm{SO}_{2}$ concentration data were the following.

Data concerning each of the 74 industrial sources (distributed in the Industrial Area shown in Fig. 1 were directly obtained by 1971 National Census figures. To give an idea of the overall emission in the region, the

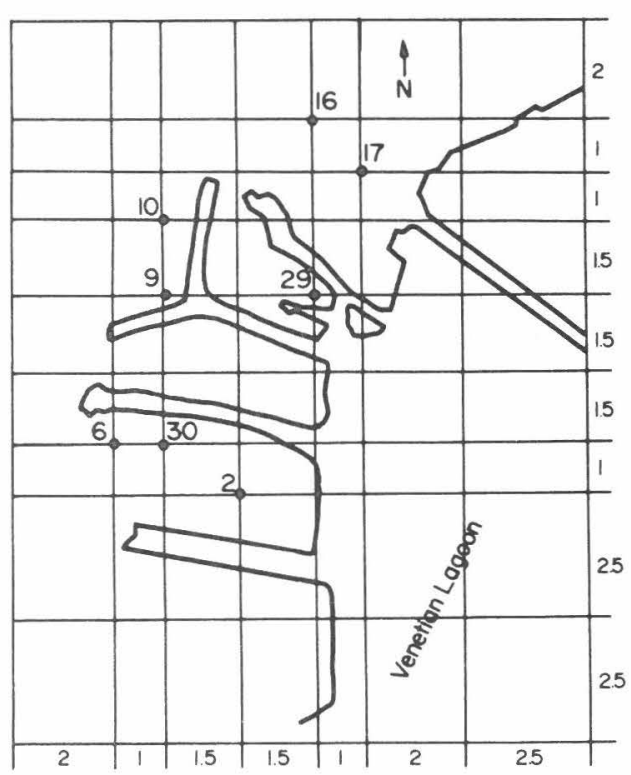

Fig. 2. The area considered for the analysis and the discretization grid at ground level. estimated pollutant amounts to about 160,000 tons per year, in addition to approximately 10,000 tons per year due to house-heating.

Both meteorological and concentration data used in the present study have been provided by the monitoring network (see Fig. 1) installed by Tecneco on behalf of the Governmental Department of Health. This network consists of one meteorological station and $24 \mathrm{SO}_{2}$ monitoring sensors.

The meteorological station, $15 \mathrm{~m}$ above the ground, records hourly wind speed and direction, temperature, pressure, humidity, rainfall, cloudiness and fog. Wind direction is recorded according to the eight sectors of the compass, thus introducing an indetermination of $\pm 22^{\circ} 30^{\prime}$. By means of wind and cloudiness data supplied by the station, Pasquill stability class (a relevant input of the $\mathrm{SO}_{2}$ concentration predictor described in Section 4) can be assigned for each hour (Pasquill, 1976).

Concentration data recorded by the 24 monitoring sensors are transmitted to a small computer which elaborates the data and records the hourly average values as well as daily statistics. In 1973, the year to which this study refers, only ten stations were put into operation. Two of them are located in Venice, the remainder are considered in this application. In general terms concentration data exhibit satisfactory reliability, while this is not true for the other types of data. In fact emission data are only average rates, a very rough input when modelling an episode, partly or mainly due to extra-release. As for meteorological measurements, one station cannot obviously point out the spatial variation of wind and diffusion parameters. Just these input uncertainties explained the poor performance of the advection-diffusion model and the subsequent reformulation in stochastic terms (see Section 4). 


\section{REAL-TIME FORECAST OF THE METEOROLOGICAL INPUTS OF THE CONCENTRATION PREDICTOR}

As pointed out in the next section, at each time step, namely at the beginning of each hour, it is necessary to forecast separately future Pasquill stability classes and wind fields, in order to supply the predictor of $\mathrm{SO}_{2}$ concentrations with all the required inputs. Specifically, such forecast of meteorological variables are made by the following simple mathematical predictors.

\section{Forecast of Pasquill stability class}

Let:

$\Delta T=1$ hour;

$k$ = time index $(k=1,2, \ldots)$;

$s(k)=$ Pasquill stability class in the $k$-th hour, namely in the interval $[(k-1) \Delta T$, $k \Delta T)$;

$h(k)=$ hour of the day corresponding to the $k$-th hour (thus, $h(k)=1,2, \ldots, 24$ ).

The following probabilistic predictor of the stability class has been used:

$$
\hat{s}(k+f \mid k) \rightarrow \max _{s(k+f)}[\operatorname{Prob}(s(k+f) \mid s(k), h(k+f))]
$$$$
f=1,2,3,4
$$

where:

$\hat{s}(k+f \mid k)=$ forecast of the stability class for the $(k+f)$-th hour, made at the end of the $k$-th hour, namely at time $k \Delta T$.

Prob $(s(k+f) \mid s(k), \quad h(k+f))=$ probability of having class $s(k+f)$ in the $(k+f)$-th hour, given the information that the class has been $s(k)$ in the $k$-th hour (namely in the hour immediately before the instant $k \Delta T$, when the forecast is made) and that the $(k+f)$-th hour is hour $h$ of the day.

In conclusion, the prediction criterion (1) corresponds to forecast the class (among the six defined by Pasquill) which maximizes the conditioned probability:

$$
\operatorname{Prob}(s(k+f) \mid s(k), h(k+f)) .
$$

\section{Wind forecast}

In the next section the following assumptions about the wind field are made: the wind vector has everywhere a negligible vertical component, wind speed is a function only of the vertical coordinate $z$, wind direction is the same in all the points of the region under consideration.

Let:

$d(k)=$ average wind direction in the $k$-th hour;

$v_{m}(k)=$ average wind speed in the $k$-th hour at level $z=z_{m}$;

$v_{R}(k)=$ average wind speed in the $k$-th hour at level $z=z_{R}=15 \mathrm{~m}$, namely at the level of the meteorological station.

At each time step, wind direction has been forecast by a probabilistic criterion quite similar to (1):

$$
\begin{array}{r}
\partial(k+f \mid k) \rightarrow \max _{\mathrm{d}(k+f)}[\operatorname{Prob}(d(k+f) \mid d(k), h(k+f))] \\
f=1,2,3,4 \quad(2)
\end{array}
$$

where:

$\delta(k+f \mid k)=$ forecast of $d(k+f)$, made at time $k \Delta T$. As for wind speed, first a predictor of $v_{R}(k+f)$, the speed at the level of the meteorological station, has been set up. Precisely, $v_{R}(k+f)$ has been forecast by means of an ARMA predictor in accordance with the techniques recommended by Box and Jenkins (1970). In more specific terms, (see, e.g., Bonivento et al., 1978; Bonivento et al., 1980 for further details) the following procedure has been used. Wind speed has first been periodically standardized, namely the variable:

$$
v_{R}^{*}(k)=\left[v_{R}(k)-\mu(h(k))\right] / \sigma(h(k))
$$

has been considered, where:

$\mu(h(k)), \sigma(h(k))=$ mean, standard deviation of hourly wind speed in hour $h$ of the day.

Standardization (3) is a way of filtering the daily periodicity from wind speed and (see predictor (6) below) can take explicitly into account the most relevant deterministic component (the daily cycle) in the forecast of wind speed. It must also be recalled that such a component is relevant in a coastal area like the Venetian Lagoon region.

The variable $v_{R}^{*}(k)$ has been regarded as a random variable belonging to a random process $\left\{v_{R}^{*}(k)\right\}_{k}$. A model of such process in the ARMA class:

$$
\begin{aligned}
v_{R}^{*}(k+1)= & \sum_{p=1}^{p^{*}} \alpha_{p} v_{R}^{*}(k-p+1)+\varepsilon(k+1) \\
& -\sum_{q=1}^{q^{*}} \beta_{q} \varepsilon(k-q+1)
\end{aligned}
$$

has been sought, where:

$$
\alpha_{p}, \beta_{q}=\text { ARMA model parameters; }
$$

$\{\varepsilon(k)\}_{k}=$ zero-mean purely random process (white noise), namely random process having the following correlation structure $(\operatorname{Exp}()=$. expectation operator $)$

$$
\operatorname{Exp}[\varepsilon(k) \varepsilon(k+\tau)] \begin{cases}=0 & \tau \neq 0 \\ \neq 0 & \tau=0\end{cases}
$$

By applying a maximum likelihood fitting technique (see, e.g., Kashyap and Rao, 1976; Goodwin and Payne 1977), to the Venetian wind speed data the most suitable ARMA model has turned out to be AR (3) (Auto Regressive of order 3), namely:

$$
v_{R}^{*}(k+1)=\sum_{p=1}^{3} \alpha_{p} v_{k}^{*}(k-p+1)+\varepsilon(k+1) .
$$

The predictor derived from model (4) is given by (see Box and Jenkins, 1970):

$$
\begin{aligned}
\hat{v}_{R}^{*}(k+1 \mid k)= & \alpha_{1} v_{R}^{*}(k)+\alpha_{2} v_{R}^{*}(k-1) \\
& +\alpha_{3} v_{R}^{*}(k-2) \\
\hat{v}_{R}^{*}(k+2 \mid k)= & \alpha_{1} \hat{v}_{R}^{*}(k+1 \mid k)+\alpha_{2} v_{R}^{*}(k) \\
& +\alpha_{3} v_{R}^{*}(k-1)
\end{aligned}
$$




$$
\begin{aligned}
\hat{v}_{R}^{*}(k+3 \mid k)= & \alpha_{1} \hat{v}_{R}^{*}(k+2 \mid k)+\alpha_{2} \hat{v}_{R}^{*}(k+1 \mid k) \\
& +\alpha_{3} v_{R}^{*}(k) \\
\hat{v}_{R}^{*}(k+4 \mid k)= & \alpha_{1} \hat{v}_{R}^{*}(k+3 \mid k)+\alpha_{2} \hat{v}_{R}^{*}(k+2 \mid k) \\
& +\alpha_{3} \hat{v}_{R}^{*}(k+1 \mid k) .
\end{aligned}
$$

By anti-standardization the wind speed predictor corresponding to Equation (5) is (see Equation (3)):

$$
\begin{aligned}
\hat{v}_{R}(k+f \mid k) & =\mu(h(k+f))+\sigma(h(k+f)) \hat{v}_{R}^{*}(k+f \mid k) \\
\text { for } f & =1,2,3,4 .
\end{aligned}
$$

From the forecast (6) of speed at the level of the meteorological station, the remainder of the wind speed field has simply been predicted by the power law:

$$
\hat{v}_{m}(k+f \mid k)=\hat{v}_{R}(k+f \mid k)\left(z_{m} / z_{R}\right)^{\gamma(3(k+f \mid k))}
$$

where the exponent is evaluated through the function reported in Table 1, first column.

In general terms, meteorological predictors (1), (2), (7) seem all rather gross and thus, together with the above mentioned simplifying assumptions on the structure of the wind field, introduce a component of uncertainty in the concentration predictor described below. However, as pointed out in the last section, such an error component is not very significant, namely the reduction of performance of the concentration predictor, due to the imperfections of the meteorological forecast, is not very important.

\section{THE KALMAN REAL-TIME PREDICTION OF FUTURE CONCENTRATION}

The procedure for setting up a real-time $\mathrm{SO}_{2}$ concentration predictor is substantially the same used by Bankoff and Hanzevack (1975). In brief, it consists of the following logical and operational steps.

(a) Solve the partial differential equation, which describes the advection and diffusion of the pollutant, by means of a numerical integration algorithm.

(b) Recognise that such an algorithm can be written in the compact form of a vector difference equation, describing the time dynamics of the entire concentration field (i.e., the vector of the concentrations at all points of the discretization grid of the numerical integration algorithm).

(c) Transform such an equation into a vector sto- chastic difference equation by introducing a proper random term ("process noise") which accounts for the overall error (= discrepancies between the actual dynamics of the concentration field and the dynamics given by the vector difference equation).

(d) Consider the measurement equation, which relates measured concentrations (by the monitoring network) to the concentration field.

(e) Transform the equation into a stochastic one, by introducing another random term ("measurement noise") which accounts for all measurement errors. (f) From the stochastic equations mentioned in (c) and (e) derive a predictor of the concentration field in accordance with the well-known Kalman forecast (see, e.g., Kalman, 1960; Jazwinski, 1971).

Procedure (a)-(f) is now illustrated step by step (for further details see Runca et al., 1979; Fronza et al., 1979). For simplicity, the procedure will be described with respect to an easier problem, namely with respect to a particular two-dimensional single-source form of the advection-diffusion equation. However (see Section 6), the extension to the three-dimensional multisource case, which was actually considered in the Venetian lagoon study is straightforward.

(a) Numerical solution algorithm of the advection -diffusion equation

The above mentioned two-dimensional single source form of the advection-diffusion equation is the following:

$$
\frac{\partial c}{\partial t}+v(z) \frac{\partial c}{\partial x}=\frac{\partial}{\partial z}\left[K^{z}(z) \frac{\partial c}{\partial z}\right]+S \delta\left(z-h_{\mathrm{eff}}\right) \delta x
$$

with initial and boundary conditions:

$$
\begin{array}{ll}
c(x, z, 0)=0 & \\
c\left(x_{W}, z, t\right)=c\left(x_{E}, z, t\right)=0 & \forall z, \forall t \\
K^{z} \frac{\partial c}{\partial z}=0 \quad z=0, h_{i n v} & \forall x, \forall t
\end{array}
$$

where:

$$
\begin{aligned}
& c=\mathrm{SO}_{2} \text { concentration. } \\
& h_{\text {eff }}=\text { effective height of the source. } \\
& h_{\text {inv }}=\text { height of the inversion layer base. } \\
& v(z) \quad=\text { wind speed (blowing in the } x \text {-direction) } \\
& \text { at level } z .
\end{aligned}
$$

Table 1. Wind and diffusion parameters versus Pasquill stability class

\begin{tabular}{ccccc}
\hline$s(t)$ & $\gamma(s(t))$ & $\rho(s(t))$ & $\begin{array}{c}K_{R}^{z}\left(z_{R}, s(t)\right) \\
\mathrm{m}^{2} \mathrm{~s}^{-1}\end{array}$ & $\begin{array}{c}K^{x}(s(t))=K^{y}(s(t)) \\
\mathrm{m}^{2} \mathrm{~s}^{-1}\end{array}$ \\
\hline$A$ & 0.05 & 6 & 45.0 & 250.0 \\
$B$ & 0.1 & 6 & 15.0 & 100.0 \\
$C$ & 0.2 & 4 & 6.0 & 30.0 \\
$D$ & 0.3 & 4 & 2.0 & 10.0 \\
$E$ & 0.4 & 2 & 0.4 & 3.0 \\
$F$ & 0.5 & 2 & 0.2 & 1.0 \\
\hline
\end{tabular}


$K^{z}(z) \quad=$ vertical diffusion coefficient at level $z$.

$x_{W} \quad=$ abscissa of western boundary of the integration region (just west of the source).

$x_{E} \quad=$ abscissa of the eastern boundary.

$S \quad=$ source emission rate.

$\delta() \quad=$. Dirac's function.

In (8)-(11) input (source and wind speed) and parameter (diffusion coefficient) dependence upon time has been neglected in order to simplify the notation in the description of the solution algorithm.

Equation (8) has been solved by means of a numerical scheme, which can be summarized as follows: it is a fractional step algorithm (see for instance Yanenko, 1971), which integrates the advection term via Carlson's method and the diffusion term via the Crank-Nicolson procedure.

Specifically, according to this approach, Equation (8) is first split into three differential equations, which separately take into account the contributions of source, advection and diffusion terms respectively:

$$
\begin{gathered}
\frac{\partial c}{\partial t}=S \delta\left(z-h_{\mathrm{eff}}\right) \delta(x) \\
\frac{\partial c}{\partial t}+v(z) \frac{\partial c}{\partial z}=0 \\
\frac{\partial c}{\partial t}=\frac{\partial}{\partial z}\left(K^{z}(z) \frac{\partial c}{\partial z}\right) .
\end{gathered}
$$

Introduce the grid notation: $\Delta x_{i}=$ grid spacing between points $(i, m)$ and $(i+1, m), \Delta z_{m}=$ grid spacing between $(i, m)$ and $(i, m+1)$. Index $i$ takes values 0,1 , $\ldots, I+1$, in particular $(1, m s)$ coincides with the source.

The time step of the numerical integration algorithm for solving Equations (12)-(14) is usually shorter than one hour. Then, let $\Delta t$ denote such a time step and let $t$ be the corresponding time index. So $c_{i, m}^{r}$ will represent concentration at time $r \Delta t$ at point $(i, m)$.

Index $m$ takes values $0,1, \ldots, M+1$. In particular ground level and mixing height correspond to $m=1$ and $m=M$ respectively. Moreover $m=0$ and $m=M+1$ represent fictitious layers, which allow boundary condition (11) to be satisfied by setting $\Delta z_{0}$ $=\Delta z_{1}, \Delta z_{M-1}=\Delta z_{M}, K_{0}^{z}=K_{1}^{z}, K_{M-1}^{z}=K_{M}^{z}, c_{i, 0}^{r}$ $=c_{i, 1}^{r}, c_{i, M}^{r}=c_{i, M+1}^{r}, \forall r, \forall i$.

Then consider the following algorithm for integrating Equations (12)-(14) in the $r$-th time interval.

Contribution of the source term (solution of Equation (12)). Solving Equation (12) simply consists of adding the contribution of the source to the concentration field at time $r \Delta t$. Precisely the step is given by:

$$
\begin{aligned}
c_{1, m s}^{*} & =c_{1, m s}^{r}+4 S \Delta t /\left(\Delta x_{1}+\Delta x_{0}\right)\left(\Delta z_{m s}+\Delta z_{m s-1}\right) \\
c_{i, m}^{*} & =c_{i, m}^{r} \text { for }(i, m) \neq(1, m s)
\end{aligned}
$$

where $c_{i, m}^{*}$ denotes the concentration field after the step.

Contribution of the advection term (solution of
Equation (13)). First consider the forward time, backward space finite difference approximation to Equation (13):

$$
c_{i, m}^{* *}=c_{i, m}^{*}-\frac{v_{m} \Delta t}{\Delta x_{i}}\left(c_{i, m}^{*}-c_{i-1, m}^{*}\right)
$$

where $c_{i, \mathrm{~m}}^{* *}$ is the concentration field after the step. Scheme (15) has first order accuracy and the Courant condition for numerical stability requires:

$$
v_{m} \Delta t<\Delta x_{i}
$$

To first order, Equation (15) is an approximation of:

$$
\frac{\partial c}{\partial t}+v_{m} \frac{\partial c}{\partial x}=\frac{v_{m}}{2}\left(\Delta x_{i}-v_{m} \Delta t\right) \frac{\partial^{2} c}{\partial x^{2}} .
$$

After many time steps, the numerical errors associated to Equation (15) can be quantified by the pseudo-diffusion coefficient:

$$
\mathscr{P}=\frac{v_{m}}{2}\left(\Delta x_{i}-v_{m} \Delta t\right)
$$

which reduces to zero for:

$$
v_{m} \Delta t=\Delta x_{i} .
$$

Since the wind is a function of the vertical coordinate, namely $v_{m}$ is not constant with $m$, in view of (16), condition (17) can be satisfied only at the height where wind speed is maximum. On the other hand, $\mathscr{P}$ should obviously be zero at least at source height $(m=m s)$ where the highest concentration gradients occur.

A solution to this problem has been provided by Carlson (e.g. Richtmyer and Morton, 1967). He suggests to use an implicit formulation instead of Equation (15), when $v_{m} \Delta t \geqslant \Delta x_{i}$. Specifically, according to Carlson's approach, if $v_{m} \Delta t \geqslant \Delta x_{i}$, Equation (15) is replaced by:

$$
c_{i, m}^{* *}=c_{i-1, m}^{*}-\frac{\Delta x_{i}}{v_{m} \Delta t}\left(c_{i-1, m}^{* *}-c_{i-1, m}^{*}\right) .
$$

The use of Equation (18) instead of Equation (15) for $v_{m} \Delta t \geqslant \Delta x_{i}$ makes Carlson's method unconditionally stable and allows reduction of the truncation error to zero at source height by choosing $\Delta x_{1}=v_{m s} \Delta t$.

Contribution of the diffusion term (solution of Equation (14)). Numerical approximation to Equation (14) can be obtained by the method due to Crank and Nicolson (1947):

$$
c_{i, m}^{r+1}=c_{i, m}^{* *}+\frac{1}{2}\left\{D_{z}\left[c_{i, m}^{r+1}\right]+D_{z}\left[c_{i, m}^{* *}\right]\right\}
$$

where

$$
\begin{aligned}
D_{z}\left[c_{i, m}^{r+1}\right]= & \frac{\Delta t}{\Delta z_{m}^{2}}\left[K_{m+1}^{z} c_{i, m+1}^{r+1}\right. \\
& \left.-\left(K_{m+1}^{z}+K_{m-1}^{z}\right) c_{i, m}^{r+1}+K_{m-1}^{z} c_{i, m}^{r+1}\right]
\end{aligned}
$$

is the standard difference operator for centered diffusion and $c^{r+1}$ denotes the concentration field at time $(r+1) \Delta t$. The overall algorithm can be summed up as: 


$$
\begin{aligned}
& c_{1, m s}^{*}= c_{1, m s}^{r}+4 S \Delta t /\left(\Delta x_{1}\right. \\
&\left.+\Delta x_{0}\right)\left(\Delta z_{m s}+\Delta z_{m s-1}\right) \quad\left(20^{\prime}\right) \\
& c_{i, m}^{*}= c_{i, m}^{r} \quad(i, m) \neq(1, m s) \\
& c_{i, m}^{* *}\left\{\begin{aligned}
= & c_{i, m}^{*}-\frac{v_{m} \Delta t}{\Delta x_{i}}\left(c_{i, m}^{*}-c_{i-1, m}^{*}\right) ; \\
& v_{m} \Delta t<\Delta x_{i} \\
= & c_{i-1, m}^{*}-\frac{\Delta x_{i}}{v_{m} \Delta t}\left(c_{i-1, m}^{* *}-c_{i-1, m}^{*}\right) ; \\
& v_{m} \Delta t \geqslant \Delta x_{i} \\
c_{i, m}^{r+1}= & c_{i, m}^{* *}+\frac{1}{2}\left\{D_{z}\left[c_{i, m}^{r+1}\right]\right. \\
& \left.+D_{z}\left[c_{i, m}^{* *}\right]\right\}
\end{aligned}\right.
\end{aligned}
$$

The effectiveness of algorithm (20)-(22) has already been discussed in detail elsewhere (Runca et al., 1979). In particular, it must be here recalled that there are other fractional step procedures illustrated in the air pollution literature (see, e.g., Shir and Shieh, 1974; Bankoff and Hanzevack, 1975). The basic difference between such procedures and algorithm (20)-(22) is that they treat only the vertical terms by an implicit formulation, while the remaining steps are solved via explicit approach. Hence the horizontal grid spacing, limited by stability conditions, is uniform and subsequently the computational effort is higher. This choice is justified by the uniform distribution of the sources (domestic sources instead of the industrial ones considered in the present study).

Apart from conspicuous computational saving, a non-uniform grid in the horizontal plane allows one to locate grid points at monitoring stations, thus avoiding any interpolation when comparing forecast and observed data.

(b) Transformation of the integration scheme into a vector difference equation

Let: $\begin{aligned} c_{i, m}(k)= & \text { average concentration at point }(i, m) \\ & \text { during the } k \text {-th hour. }\end{aligned}$

$e_{i, m}(k)=0$ for $(i, m) \neq(1, m s)$

$e_{1, \mathrm{~ms}}(k)=4 S(k) \Delta T /\left(\Delta x_{1}+\Delta x_{0}\right)\left(\Delta z_{m s}+\Delta z_{m s-1}\right)$

$S(k)=$ emission during the $k$-th hour.

Moreover, in the following a notation like $\left|b_{i, m}(k)\right|$ for $i=0,1, \ldots, I+1 ; m=0,1, \ldots, M+1$, will denote the vector $\mid b_{0,0}(k) b_{1,0}(k) \ldots \ldots, b_{I+1,0}(k) b_{0,1}(k) \ldots$ $\left.b_{I+1, M+1}(k)\right|^{T}(T=$ vector transposition symbol).

Then, it can be shown that algorithm (20)-(22) leads to the following vector difference equation, which is a compact description of the hourly dynamics of the entire concentration field:

$$
\begin{aligned}
X(k+1)= & \phi(v(k+1), s(k+1)) X(h) \\
& +\phi(v(k+1), s(k+1)) E(k+1)
\end{aligned}
$$

where:

$$
\begin{aligned}
& X(k)=\left|c_{i, m}(k)\right| ; \\
& E(k)=\left|e_{i, m}(k)\right| ; \\
& v(k)=\left|v_{m}(k)\right| ; \\
& \phi(v(k), s(k))=\text { suitable matrix, only depending upon } \\
& \text { the wind field } v(k) \text { and Pasquill } \\
& \text { stability class } s(k) .
\end{aligned}
$$

A formal (though slightly simplified) proof of the above statement can be found in Fronza et al. (1979). Equation (23) is there derived by a long and cumbersome (though conceptually simple) development, which is not reported here for brevity. Anyway, the transformation of Equations (20)-(22) into Equation (23) is a well-known property in numerical calculus literature. However, according to our experience, some lack 'of explanation by Bankoff and Hanzevack (1975) on this formal point has partially limited the acknowledgement of their fundamental contribution.

(c) Transformation of Equation (23) into a stochastic vector difference equation

Such transformation simply consists of adding a random term to the right-hand side of Equation (23):

$$
\begin{aligned}
X(k+1)= & \phi(v(k+1), s(k+1)) X(k) \\
& +\phi(v(k+1), s(k+1)) E(k+1) \\
& +n(k)
\end{aligned}
$$

where:

$n(k)=$ stochastic term ("process noise"), which accounts for all the sources of disagreement between the model and the actual dynamics of the pollution phenomenon (i.e., for neglected physical inputs in the advectiondiffusion equation such as rain or chemical reactions, for errors introduced by the assignment of parameter values, for errors due to the model structure, to numerical inaccuracies and so on).

The random process $\{n(k)\}_{k}$ is commonly assumed to be zero-mean white noise, namely to have a correlation structure:

$$
\operatorname{Exp}\left[n(k) n^{T}(k+\tau)\right]= \begin{cases}Q(k) & \tau=0 \\ 0 & \tau \neq 0\end{cases}
$$

(d) Measurement relationship

Let:

$y(k)=$ vector of average concentration measurements in the $k$-th hour (taken in monitoring stations all coincident with grid points).

Then the relationship between $y(k)$ and the concentration field $X(k)$ is:

$$
y(k)=H X(k)
$$

where $H$ is a suitable matrix, whose elements are either 0 or 1 .

(e) Transformation of the measurement relationship into a stochastic one

Such transformation simply consists of introducing a random term into Equation (4):

$$
y(k)=H X(k)+w(k)
$$

where:

$w(k)=$ stochastic term ("measurement noise") which accounts for all errors in measurements.

Also the process $\{w(k)\}_{k}$ is assumed to be zero-mean white noise: 


$$
\operatorname{Exp}\left[w(k) w^{T}(k+\tau)\right]= \begin{cases}R(k) & \tau=0 \\ 0 & \tau \neq 0\end{cases}
$$

\section{( $f$ ) Kalman predictor of concentrations}

The pair of relationships (23')-(24b), here rewritten for commodity:

$$
\begin{aligned}
X(k+1)= & \phi(v(k+1), s(k+1)) X(k) \\
& +\phi(v(k+1), s(k+1)) E(k+1) \\
& +n(k) \\
y(k)= & H X(k)+w(k)
\end{aligned}
$$

represents a "stochastic dynamical system" from which real-time pollution forecast can be derived, namely a recursive relationship which, at the beginning of each time step, predicts future $\mathrm{SO}_{2}$ concentrations on the basis of current information about concentrations, emission and meteorology. Specifically, the recursive one-hour ahead forecast algorithm (Kalman predictor) derived from Equations 25(a) and (b) is given by (see, e.g., Jazwinski, 1970):

$$
\begin{aligned}
\hat{X}(k \mid k)= & \hat{X}(k \mid k-1)+G(k)[y(k) \\
& -H \hat{X}(k \mid k-1)] \\
\hat{X}(k+1 \mid k)= & \phi(\hat{v}(k+1 \mid k), \hat{s}(k+1 \mid k)) \hat{X}(k \mid k) \\
& +\phi(\hat{v}(k+1 \mid k), \hat{s}(k+1 \mid k)) E(k+1)
\end{aligned}
$$

where:

$\hat{X}(k+1 \mid k)=$ prediction of $X(k+1)$ made at time $k \Delta T$ namely at the end of the $k$-th interval of time.

$\hat{X}(k \mid k)=$ filtered state, namely a posteriori (i.e. at time $k \Delta T)$ estimation of $X(k)$ on the basis of the new available datum $y(k)$. Precisely such an estimation is given by Equation (26) as a correction of the previous forecast $\hat{X}(k \mid k-1)$ and is introduced in Equation (27) instead of $\hat{X}(k \mid k-1)$ in order to give a better prediction of $X(k+1)$.

$$
\begin{aligned}
G(k)=\quad & P(k \mid k-1) H^{T}\left[H P(k \mid k-1) H^{T}\right. \\
& +R(k)]^{-1} \\
& \text { is Kalman gain }
\end{aligned}
$$

The $f$-hour ahead prediction $(f=2,3,4)$ is obtained recursively by:

$$
\begin{aligned}
\hat{X}(k+f \mid k)= & \phi(\hat{v}(k+f \mid k), \hat{s}(k+f \mid k)), \hat{X}(k+f-1 \mid k) \\
& +\phi(\hat{v}(k+f \mid k), \hat{s}(k+f \mid k)) E(k+f)
\end{aligned}
$$

Note that (as stated in the Introduction), the inputs required by the concentration predictor (26)-(31) are the forecast of wind field $\hat{v}(k+f \mid k)$ and stability class $\hat{s}(k+f \mid k)$ (given in the previous section), as well as scheduled emissions $E(k+f)$. Of course, when turning from the two-dimensional case considered here to the three dimensional situation of the Venetian case (see last section) also wind direction forecast is required. Moreover, the actual implementation of predictor (26)-(31) raises a number of conceptual and operational problems (evaluation of $Q(k)$ and $R(k)$, computational effort, etc.) which are also mentioned in the last section.

\section{THE REAL-TIME EMISSION CONTROL POLICY}

\section{(a) Policy statement}

Turn again to the multisource-three dimensional Venetian case and consider the corresponding Kalman concentration predictor of the type (26)-(31). Let $d(d=1,2, \ldots, D)$ denote the source index.

First, it has been assumed that, at each source, the actual settling of the control action takes one hour. Precisely, an operation of fuel replacement decided at time $k \Delta T$ is effective only since $(k+1) \Delta T$, namely it displays its effects as emission reduction only in the $(k+2)$-nd hour. In view of such an assumption (which is commented on below), a control action decided at time $k \Delta T$ consists of a reduction of $S^{d}(k+2)(d=1$, $2, \ldots, D)$, the emission scheduled by the $d$-th source for the $(k+2)$-nd hour, and not of a reduction of $S^{d}$ $(k+1)$.

The control policy is specified as follows. Let $(f=2$, $3,4)$ :

$\hat{x}_{M}(k+f \mid k)=$ maximum ground level hourly concentration, forecast at time $k \Delta T$ by the Kalman concentration predictor for the $(k+f)$-th hour;

$$
\Delta_{M}(k+f \mid k)=\left\{\begin{array}{cc}
{\left[x_{M}(k+f \mid k)-A\right] / \hat{x}_{M}(k+f \mid k)} & \hat{x}_{M}(k+f \mid k)>A \\
0 & \hat{x}_{M}(k+f \mid k) \leqslant A
\end{array}\right.
$$

$$
\begin{aligned}
P(k \mid k-1)= & \operatorname{Exp}[(\hat{X}(k \mid k-1)-X(k))(\hat{X}(k \mid k-1) \\
& \left.-X(k))^{T}\right]=\text { forecast error covari- } \\
& \text { ance matrix. }
\end{aligned}
$$

In turn, such covariance matrix is recursively evaluated through the following equations:

$$
\begin{aligned}
P(k \mid k)= & P(k \mid k-1)\left\{I-H^{T}\left[H P(k \mid k-1) H^{T}\right.\right. \\
& \left.+R(k)]^{-1} H P(k \mid k-1)\right\} \\
P(k+1 \mid k)= & \phi(\hat{v}(k+1 \mid k), \hat{s}(k+1 \mid k)) P(k \mid k) \\
& \times \phi(\hat{v}(k+1 \mid k), \hat{s}(k+1 \mid k))+Q(k+1)(30)
\end{aligned}
$$

$A=$ preassigned reference concentration value. Then assume the following control action at each time step: reduce the emission $S^{d}(k+2)$, scheduled for the $(k+2)$-nd hour in source $d$, by a percentage $\theta(k+2)$ given by:

$$
\theta(k+2)=\sum_{f=2}^{4} \lambda_{f} \Delta_{M}(k+f \mid k)
$$

In Equation (33), $\lambda_{f}$ are preassigned weights such that $0 \leqslant \lambda_{f} \leqslant 1$ and moreover $\lambda_{2}+\lambda_{3}+\lambda_{4}=1$. Therefore, 
since $0 \leqslant \Delta_{M}(k+f \mid k)<1$ in view of Equation (32), from Equation (33) it always turns out $0 \leqslant \theta$ $(k+2)<1$ (actually, the range of $\theta(k+2)$ is narrower in view of the production constraint shown below).

Control policy (33) is explained and completed by the following comments.

In view of Equation (33), $\theta(k+2)$ is nonzero whenever (at least) one of the three $\Delta_{M}(k+f \mid k)$ is greater than zero $(f=2,3,4)$. Namely (see Equation (32)), a reduction of $S^{d}(k+2)$ is requested (via fuel replacement starting at $k \Delta T$, in view of the delay due to operation times) whenever (at least) one forecast of maximum concentration, made at time $k \Delta T$, exceeds the reference level $A$.

The forecast $\hat{x}_{M}(k+2 \mid k)$ by the Kalman predictor is obviously more reliable than $\hat{x}_{M}(k+3 \mid k)$, which, in turn, is more reliable than $\hat{x}_{M}(k+4 \mid k)$. Thus, these three forecasts must affect the decision $\theta(k+2)$ by different weights in equation (33), i.e., it is reasonable to set $\lambda_{2}>\lambda_{3}>\lambda_{4}$.

The control action $\theta(k+2)$ given by Equation (33) does not depend upon the source index $d$, i.e. the percent reduction is the same for all sources. This uniformity may appear too gross a simplification, since the detail of information supplied by the Kalman concentration predictor seems to allow a selective control of the sources. However, in the Venetian case (see the comment at the end of Section 2) the reliability of data concerning the scheduled emissions has not justified more sophisticated control policies.

The forecast horizon for the Kalman predictor and control action (33) has been taken as $4 \mathrm{~h}$ while the delay due to operation ( = fuel replacement) times has been assumed $1 \mathrm{~h}$. Naturally, by proper index modifications in Equations (32), (33), the analysis might be extended to longer forecast horizons as well as to longer operation times. Of course, prediction reliability may become poor as the forecast horizon gets longer.

In view of Equations (32) and (33), the decision $\theta(k+2)$ is based on forecast maximum ground level concentrations. However, the forecast of different variables (e.g. spatial averages) can be taken into account as the guide for deciding the control action.

(b) Actual implementation of the control action and extra-cost evaluation

In this subsection the operation of fuel replacement, necessary in order to obtain the emission reduction $\theta$ $(k+2)$ is specified in terms of required fuel quantities.

In the following the indexes HS (High Sulphur) and LS (Low Sulphur) will respectively denote the standard fuel used by all the polluters and the cleaner fuel required for the control action.

First, if $t_{\mathrm{HS}}$ and $t_{\mathrm{LS}}$ denote the sulphur contents of the two fuels, the quantities (in tons) $q_{\mathrm{HS}}^{d}(k+2)$ and $q_{\mathrm{LS}}^{d}$ $(k+2)$ to be burnt in the $(k+2)$-nd hour, must be such that:

$$
\begin{gathered}
{ }^{t}{ }_{\mathrm{HS}} q_{\mathrm{HS}}^{d}(k+2)+t_{\mathrm{LS}} q_{\mathrm{LS}}^{d}(k+2)= \\
{[1-\theta(k+2)] S^{d}(k+2)}
\end{gathered}
$$

in view of the definition itself of $\theta(k+2)$.

Moreover, note that $S^{d}(k+2) / t_{\mathrm{HS}}$ is the quantity of fuel scheduled by the $d$-th source for the $(k+2)$-nd hour. Since no change in the production of the $d$-th plant is desired, such overall quantity must not be modified by the control action, namely it must be:

$$
q_{\mathrm{HS}}^{d}(k+2)+q_{\mathrm{LS}}^{d}(k+2)=S^{d}(k+2) / t_{\mathrm{HS}}
$$

From equations (34) and (35), it is straightforward to obtain:

$$
\begin{aligned}
& q_{\mathrm{HS}}^{d}(k+2)= {\left[1-\theta(k+2)-t_{\mathrm{LS}} / t_{\mathrm{HS}}\right] } \\
& \mathrm{S}^{d}(k+2) /\left(t_{\mathrm{HS}}-t_{\mathrm{LS}}\right) \\
& q_{\mathrm{HS}}^{d}(k+2)=\theta(k+2) S^{d}(k+2) / \\
&\left(t_{\mathrm{HS}}-t_{\mathrm{LS}}\right)
\end{aligned}
$$

Note that, in view of the non-negativity of $q_{\mathrm{HS}}^{d}(k+2)$ in Equation $\left(36^{\prime}\right)$, the control action $\theta(k+2)$ is constrained to be $\leqslant\left(1-t_{\mathrm{LS}} / t_{\mathrm{HS}}\right)$. In particular, $\theta(k+2)$ $=1-t_{\mathrm{LS}} / t_{\mathrm{HS}}$ corresponds to complete replacement of the HS fuel $\left(q_{\mathrm{HS}}^{d}(k+2)=0, q_{\mathrm{LS}}^{d}=S^{d}(k+2) / t_{\mathrm{HS}}\right.$ from Equations (36)), i.e. to the "strongest" control action.

If $C_{\mathrm{HS}}$ and $C_{\mathrm{LS}}$ denote the unit costs of the two fuels, the overall fuel cost in the $(k+2)$-nd hour for the $D$ plants under control action is given by:

$$
C^{\mathrm{c}}(k+2)=\sum_{d=1}^{D}\left[C_{\mathrm{HS}} \mathrm{q}_{\mathrm{HS}}^{d}(k+2)+C_{\mathrm{LS}} q_{\mathrm{LS}}^{d}(k+2)\right]
$$

namely, in view of Equations (36') and (36"):

$$
\begin{aligned}
C^{c}(k+2)= & {\left[C_{\mathrm{HS}}\left(1-\theta(k+2)-t_{\mathrm{LS}} / t_{\mathrm{HS}}\right)\right.} \\
& \left.+C_{\mathrm{LS}} \theta(k+2)\right] S(k+2) /\left(t_{\mathrm{HS}}-t_{\mathrm{LS}}\right)
\end{aligned}
$$

where: $S(k+2)=\sum_{d=1}^{D} S^{d}(k+2)$ is the overall scheduled emission.

The fuel cost without control action would simply be:

$$
C^{n c}(k+2)=C_{\mathrm{HS}} S(k+2) / t_{\mathrm{HS}}
$$

hence the percent extra-cost due to the control action is:

$$
\psi(k+2)=\left[C^{c}(k+2)-C^{n c}(k+2)\right] / C^{n c}(k+2)(39)
$$

In view of Equations (37) and (38) and of some simple but cumbersome computations it turns out to be:

$$
\psi(k+2)=\theta(k+2)\left(C_{\mathrm{LS}} / C_{\mathrm{HS}}-1\right)\left(1-t_{\mathrm{LS}} / t_{\mathrm{HS}}\right)(40)
$$

The compact formula (40) points out the dependence of the percent extra-cost upon both the intensity $\theta(k+2)$ of the emission reduction and the LS/HS unit cost and sulphur content ratios.

\section{RESULTS OF THE APPLICATION TO THE VENETIAN LAGOON CASE}

\section{Specifications concerning the integration scheme}

The region of interest $(16.5 \mathrm{~km} \times 18.0 \mathrm{~km}$ wide) has 
been discretized by means of $10 \times 12 \times 7=840$ grid points. The horizontal grid spacing ranges from a minimum of $1 \mathrm{~km}$ to a maximum of $2.5 \mathrm{~km}$ (see Fig. 2, where the monitoring stations, all coincident with grid points are also reported). The vertical grid sizes were specified as follows:

$$
\Delta z_{m}= \begin{cases}50 \mathrm{~m} & m=1,2 \\ 75 \mathrm{~m} & m=3,4 \\ \left(h_{\text {inv }}-250\right) / 2 & m=5,6\end{cases}
$$

Since no measurement of the mixing depth $h_{\text {inv }}$ was available, $h_{\mathrm{inv}}$ was kept equal to $500 \mathrm{~m}$ in all simulations. On the above mesh, the following advection-diffusion equation has been integrated (differently from Equation (8), here input and parameter dependence upon time is explicitly pointed out):

$$
\begin{aligned}
\frac{\partial c}{\partial t}+v_{x}(z, s(t)) \frac{\partial c}{\partial x}+v_{y}(z, s(t)) \frac{\partial c}{\partial y} \\
=K^{x}(s(t)) \frac{\partial^{2} c}{\partial x^{2}}+K^{y}(s(t)) \frac{\partial^{2} c}{\partial y^{2}} \\
+\frac{\partial}{\partial z}\left[K^{z}(z, s(t)) \frac{\partial c}{\partial z}\right] \\
+S(x, y, z, t)
\end{aligned}
$$

with initial and boundary conditions:

$$
\begin{aligned}
& c(x, y, z, t)=0 \quad t=0 \\
& K^{z} \frac{\partial c}{\partial z} \quad=0 \quad z=0, h_{\text {inv }} \\
& c(x, y, z, t)=0 \quad \forall t \text { at side boundaries. }
\end{aligned}
$$

In Equation (41), $S(x, y, z, t)$ is the source term, $v_{x}(z, s(t))$ and $v_{y}(z, s(t))$ are the wind speed components in the horizontal plane, $s(t)$ denotes atmospheric stability at time $t$ in accordance with the classification by Pasquill. Boundary condition (42c) is acceptable since the integration region is extended enough in the horizontal plane so that at its boundaries pollution is actually negligible. As for $K^{z}(z, s(t))$, the classical formula by Shir and Shieh (1974) has been modified in the following way:

$$
K^{z}(z, s(t))=K^{D}(s(t)) z \exp \left(-\rho(s(t)) \cdot z / h_{\text {inv }}\right)
$$

The values of $\rho(s(t))$ are reported in Table 1, second column. As for $K^{D}(s(t))$, it has been obtained by:

$$
K^{D}(s(t))=z_{R}^{-1} K_{R}^{z}\left(z_{R}, s(t)\right) \exp \left(\rho(s(t)) z_{R} / h_{\text {inv }}\right)
$$

where $K_{R}^{z}(z, s(t))$ is the vertical diffusion coefficient at the level of the meteorological station and is reported in Table 1 (third column). Finally, as for horizontal diffusion coefficients, it has been assumed $K^{x}(s(t))$ $=K^{y}(s(t))=$ values reported in Table 1 (fourth column).

\section{Specifications concerning the Kalman predictor}

All the details concerning the actual implementation of the Kalman predictor described in Section 5 are reported by Fronza et al. (1979) and are here sum- marized (see also Bankoff and Hanzevack, 1975).

The noise intensities (covariances) $Q(k)$ and $R(k)$ [see Equations (28) and (30)] are evaluated at each time step by means of the algorithm due to Jazwinski (1969).

Wrong information about scheduled emission is heuristically corrected through an a posteriori pollutant mass balance ( = comparison between filtered and previously forecast overall mass of pollutant at ground level).

The computational burden of the predictor is high (see Equations (26) and (31) and recall that $X(k)$ has 840 components). The burden has been reduced by filtering (Equation (26) of the predictor) only a subvector of $X(k)$ (i.e., precisely, the subvector of concentrations in the most polluted zones of the field).

In spite of such reduction, it has still been necessary to use a relatively big computer (IBM 370/158). Note, however, that the computational problem is only related to the choice of the predictor and not to the concept of real-time control in general (namely to scheme (i)-(iv) stated in the introduction). In other terms, if a simpler concentration predictor. (Finzi et al., 1978; Finzi et al., 1979; Finzi et al., 1980; Bacci et al., 1981) were used, the entire control procedure (i)-(iv) would require a very low computational effort.

\section{Performance of the Kalman predictor}

In non-episode situation, the predictor has given good performances, but this is usual for "reasonable" predictors and is not a particularly significant result. For episodes (see again Fronza et al., 1979 for the details), the performance has also been satisfactory. For example, consider the curves of Figs. 3(a) and 4(a), corresponding to two relevant episodes in 1973 (the year to which the present study refers). Such episodes looked particularly "difficult" from the prediction viewpoint, because high concentrations had been measured only in a very limited zone of the region (practically in one station).

The uncertainty introduced into the concentration forecast by the gross meteorological predictors described in Section 2 has also been evaluated. Precisely, the Kalman concentration predictor has also been run in correspondence with two other types of meterological inputs.

(A) Persistent meteorological inputs (future values of the meteorological variables will be equal to the current ones). This is the roughest treatment of the meteorological inputs and therefore gives the lower bound of the performance given by the Kalman concentration predictor.

(B) True meteorological inputs ( = perfect forecast of the meteorological variables). This yields the upper bound of the performance of the Kalman predictor.

The results are shown in Figs. 3(b), 3(c), 4(b) and 4(c). By comparing Fig. 3(a) with Fig. 3(c) and Fig. 4(a) with Fig. 4(c), the uncertainty due to the imperfection of the 

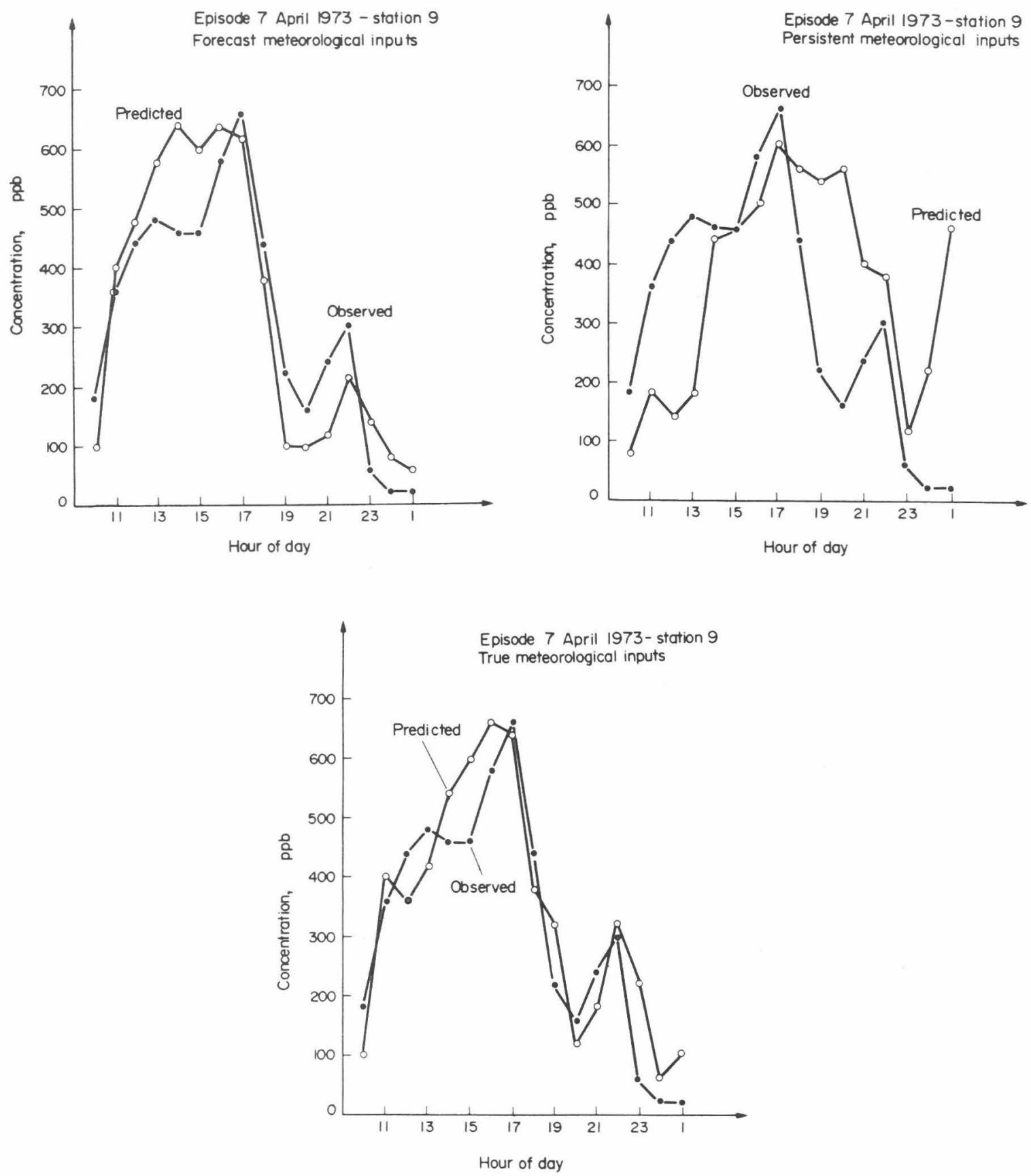

Fig. 3. Performance of the Kalman predictor under: (a) forecast, (b) persistent, (c) true meteorological inputs (episode 7 April 1973).

meteorological forecast turns out to be tolerable. In particular, the correlation between predicted and observed concentrations has been respectively $0.90,0.32$ and 0.92 for the three situations of Fig. 3 and $0.76,0.50$ and 0.77 for the three situations of Fig. 4 .

Cost-effectiveness analysis of the real-time control policy

As indicated by Equations (32) and (33), the dependence of the control action upon the reference concentration $A$ is relevant. Hence an analysis has been carried out using $A$ as a parameter.
Furthermore (see Equation (32)) two different triplets of weights have been considered, namely $\lambda^{\prime}=\{1 ; 0$; $0\}$ and $\lambda^{\prime \prime}=\{0.6 ; 0.3 ; 0.1\}$.

Finally, two types of LS fuels have been tested separately (denoted by indexes LS1 and LS2 respectively), with LS-HS sulphur content ratios:

$$
t_{\mathrm{LS} 1} / t_{\mathrm{HS}}=0.33 \quad t_{\mathrm{LS} 2} / t_{\mathrm{HS}}=0.10
$$

and LS-HS unit cost ratios:

$$
C_{\mathrm{LS} 1} / C_{\mathrm{HS}}=1.1 \quad C_{\mathrm{LS} 2} / C_{\mathrm{HS}}=1.5 .
$$

The results obtained by applying the control policy 

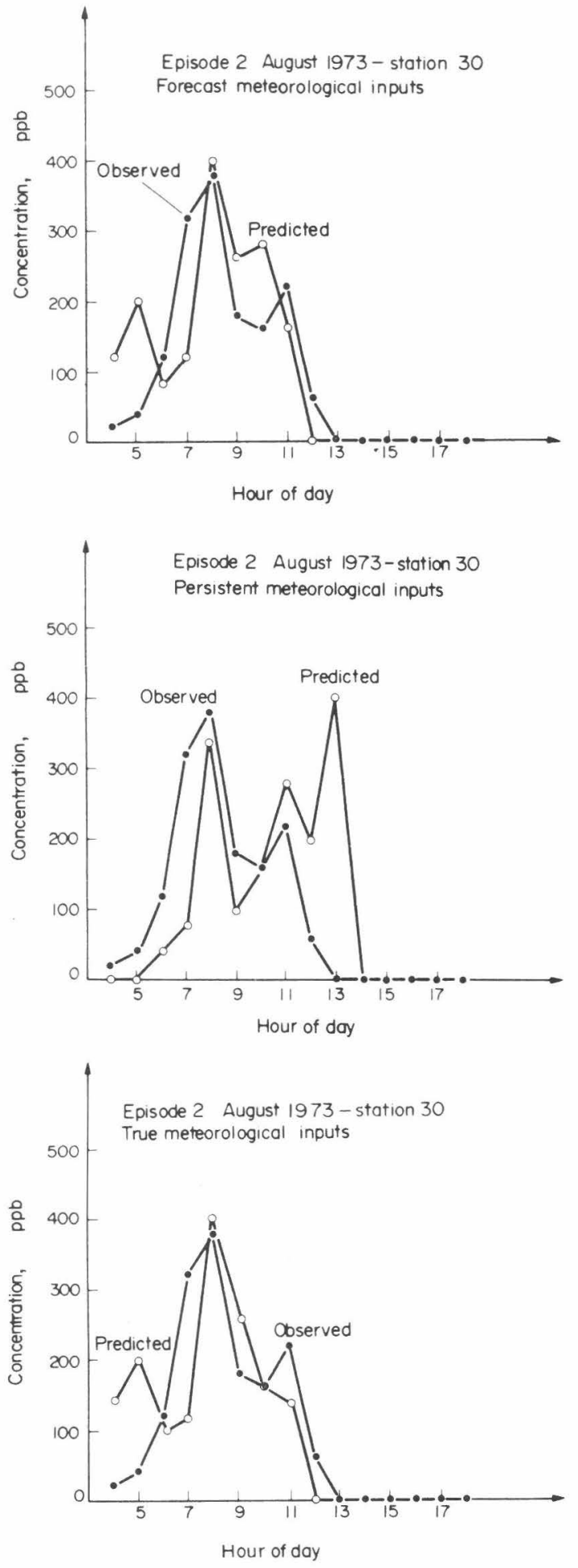

Fig. 4. Performance of the Kalman predictor under: (a) forecast, (b) persistent, (c) true meteorological inputs (episode 2 August 1973).

(described in Section 5) during April 1973 are summarized by the cost-effectiveness curves of Fig. 5 .

Specifically, the effectiveness index $I_{E}$ is defined as follows (naturally, alternative effectiveness definitions are possible). Let $x_{M}^{c}(k)$ and $x_{M}^{n c}(k)$ denote the maximum ground level concentration in the $k$-th hour of

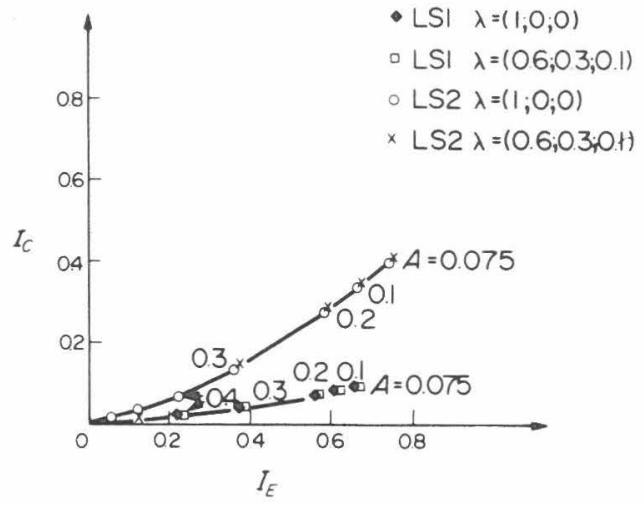

Fig. 5. Cost-effectiveness of real-time control in April 1973 for different values of the parameter $A$ (in ppm) shown by plotting cost index $\left(I_{c}\right)$ as a function of effectiveness index. $\left(I_{E}\right)$.

April 1973, respectively, with and without emission control. Moreover, consider the time averages:

$$
\bar{x}_{M}^{c}=\sum_{k} x_{M}^{c}(k) / N \quad \bar{x}_{M}^{n c}=\sum_{k} x_{M}^{n c}(k) / N
$$

where the sums are extended over the total number $N$ of hours in the month of April. Then,

$$
I_{E}=\left(\bar{x}_{M}^{n c}-\bar{x}_{M}^{c}\right) / \bar{x}_{M}^{n c}
$$

The cost index $I_{c}$ is the overall percent extra-cost due to the control action, namely:

$$
I_{c}=\sum_{k}\left[C^{c}(k)-C^{n c}(k)\right] / \sum_{k} C^{n c}(k)
$$

The first conclusion to be drawn by direct inspection of Fig. 5 is that there is no significant difference between the results of control action under the two different triplets of weights $\lambda^{\prime}=\{1,0,0\}$ ( = control only based on two-hour forecast, see Equation (33)) and $\lambda^{\prime \prime}=\{0.6 ; 0.3 ; 0.1\}(=$ control action considering also the three and four-hour predictions).

Furthermore, from Fig. 5 each effictiveness level can be reached by fuel LSl at a lower cost than by fuel LS2. Namely the assumed LS/HS sulphur content and unit cost ratios are such that the higher sulphur content of LSl is more than compensated by its lower cost.

Naturally the use of LS2 gives a higher upper bound of effectiveness ( = end abscissa of the curves of Fig. 7) than the use of LS1. Precisely, the "less clean less expensive fuel" LS 1 is sufficient to obtain effectiveness levels up to about $65 \%$, while effectiveness up to about $75 \%$ can be reached by using the more costly fuel LS2. Thus, a fuel like LS2, which is more than three times cleaner than LS1 (see Equation (43)) produces a maximum effectiveness which is only a few percent higher.

To complete the picture, some episode time patterns (for different $A$-values, $\lambda$-weights and types of fuel) are shown in Fig. 6, while the corresponding time patterns of the control action $\theta(k)$ are shown in Fig. 7. In particular, the action turns out to have an acceptably regular time profile, namely it does not consist of a sequence of undesirable control "shocks". 

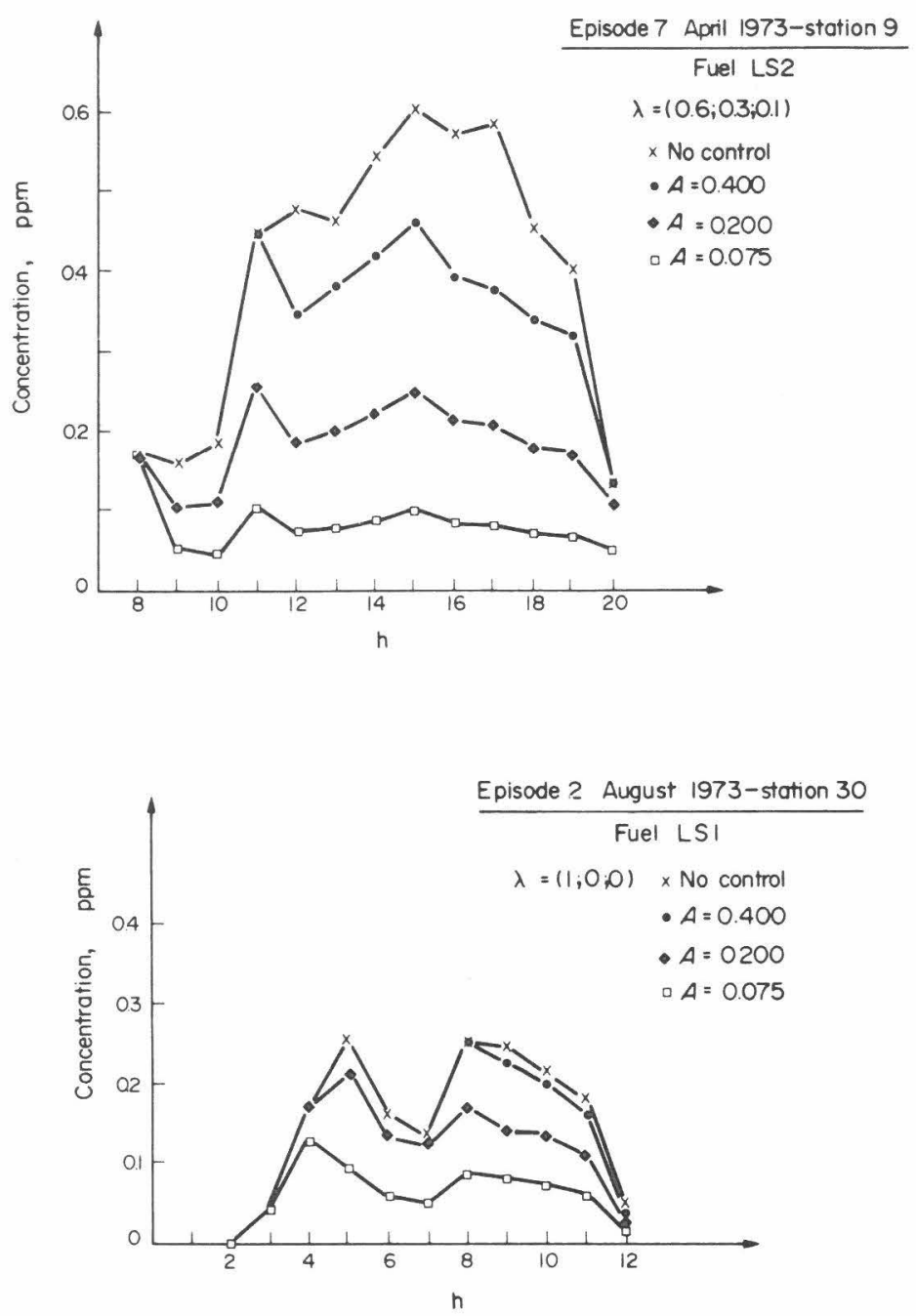

Fig. 6. Concentration time patterns for different control policies: (a) episode 7 April 1973; (b) episode 2 August 1973.
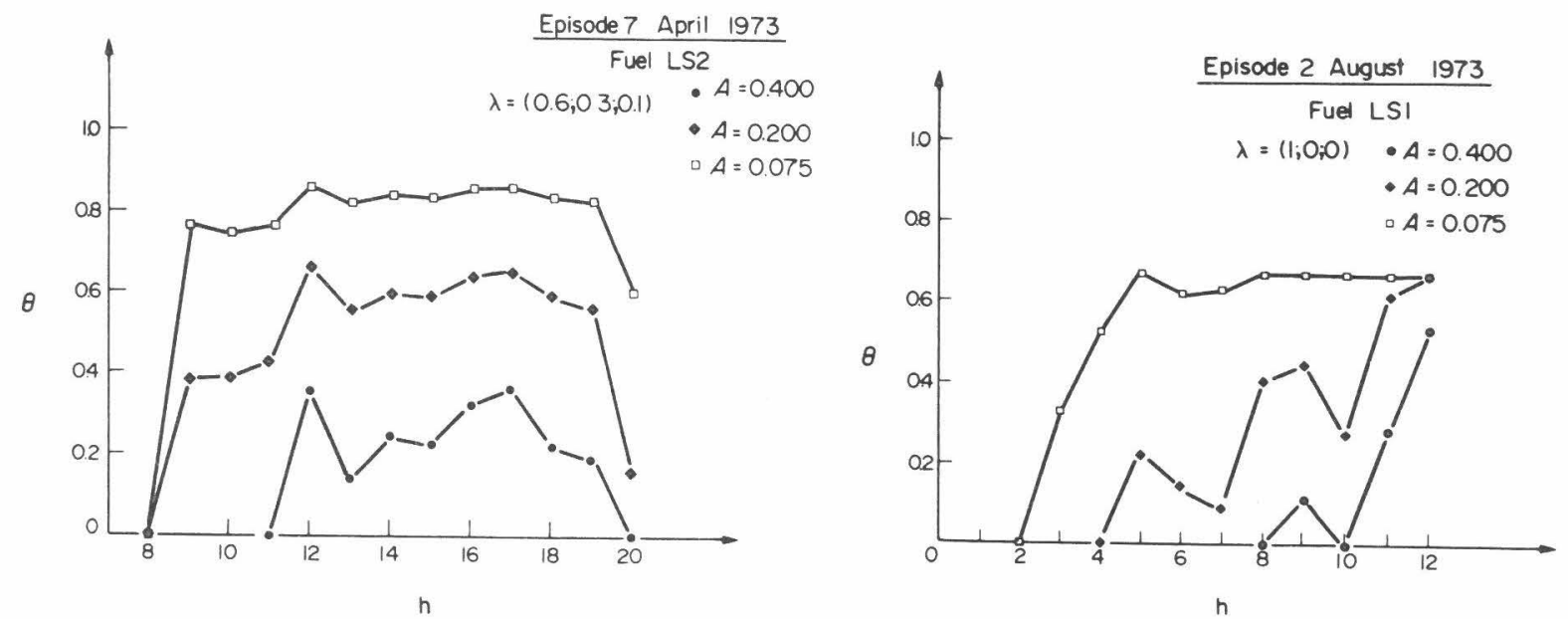

Fig. 7. Time pattern of the control action (a) episode 7 April 1973; (b) episode 2 August 1973. 


\section{REFERENCES}

Atkinson S. E. and Lewis D. H. (1974) A cost-effectiveness analysis of alternative air quality control strategies. $J$. Envir. Econ. Management 7, 237-250.

Bacci P., Bolzern P. and Fronza G. (1981) A stodiostic predictor of air pollution based on short-term meteorological forecast, J. appl. Met., 21.

Bankoff S. G. and Hanzevack E. L. (1975). The adaptive filtering transport model for prediction and control of pollutant concentration in an urban airshed. Atmospheric Environment 9, 793-808.

Barbieri D., Baroni M. and Finzi G. (1979). Multivariate real-time predictors of sulphur dioxide concentrations in Milan city, Proc. NATO-CCMS 10th Int. Technical Meeting on Air Pollution Modelling, Rome, Italy.

Bonivento C., Fronza G. and Tonielli A. (1978) Stochastic predictors of site wind. Ricerche di Automatica 9, 171-183.

Bonivento C., Fronza G. and Tonielli A. (1980) Real-time prediction of local wind speed by means of stochastic models, Proc. 14th IRCHA Colloque International sur les Atmosphères Polluées, Vert-le-Petit, France (in press).

Box G. E. P. and Jenkins G. M. (1970). Time Series Analysis, Forecasting and Control, Holden Day, San Francisco.

Crank J. and Nicolson P. (1947) A practical method for numerical solution of partial differential equations of heat conduction type, Proc. Cambridge Phil. Soc. 43, 50-67.

Emanuel W. R., Murphy B. D. and Huff D. D. (1978) Optimal siting of energy facilities for minimum air pollutant exposure on a regional scale. J. Envir. Management $7,147-155$.

Finzi G., Fronza G. and Rinaldi S. (1978). Stochastic modelling and forecast of the Dosage Area Product, Atmospheric Environment 12, 831-838.

Finzi G., Zannetti P., Fronza G. and Rinaldi S. (1979) Realtime prediction of $\mathrm{SO}_{2}$ concentrations in the Venetian lagoon case. Atmospheric Environment 13, 1249-1255.

Finzi G., Fronza G. and Spirito A. (1980) Multivariate stochastic models of sulphur dioxide pollution in an urban area. J. Air Pollut. Control Ass. 30, 1212-1215.

Fronza G., Spirito A. and Tonielli A. (1979) Real-time forecast of air pollution episodes in the Venetian region: part II: the Kalman predictor. Appl. Math. Modelling 3, 409-415.

Goodwin G. C. and Payne R. L. (1977) Dynamic System Identification: Experiment Design and Data Analysis, Academic Press, New York.

Guldmann J. and Shefer D. (1976) Stack height as a means for air quality control: a mathematical programming approach. J. Envir. Management 4, 241-249.

Jazwinski A. H. (1969) Adaptive filtering. Automatica, 5, 475-495.

Jazwinski A. H. (1970) Stochastic Processes and Filtering Theory, Academic Press, New York.

Kalman R. E. (1960) A new approach to linear filtering and prediction theory, Trans. of ASME, J. Basic Engn 82, 17 25.

Kashyap R. L. and Rao A. R. (1976) Dynamic Stochastic Models from Empirical Data, Academic Press, New York.

Leavitt J. M., Carpenter S. B., Blackwell J. P. and Montgomery T. L. (1971) Meteorological program for limiting power plant stack emissions, J. Air Pollut. Control Ass. 21, 400-405.

Marziano G. L., Shir C. C., Shieh L. J., Sutera A., Gianolio L. and Ciprian M. (1979) Study of the $\mathrm{SO}_{2}$ distribution in Venice by means of an air quality simulation model. Atmospheric Environment 13, 447-487.

Pasquill F. (1976) Atmospheric Diffusion, Van Nostrand, London.

Richtmyer R. D. and Morton J. W. (1967). Difference Methods for Initial-value Problems, Interscience, New York.

Runca R., Melli P. and Zannetti P. (1976) Computation of long-term average $\mathrm{SO}_{2}$ concentration in the Venetian Area. Appl. Math. Modelling 1, 9-15.

Runca E., Melli P. and Spirito A. (1979) Real-time forecast of air pollution episodes in the Venetian region: Part. I: the advection-diffusion model. Appl. Math. Modelling 3, 402408.

Seinfeld J. H. and Kyan C. P. (1971) Determination of optimal air pollution control strategies. Socio-Economic Planning Sci. 5, 173-190.

Shepard D. S. (1970) A load shifting model for air pollution control in the electric power industry, J. Air Pollut. Control Ass. 20, 756-761.

Shir C. C. and Shieh L. J. (1974) A generalized urban air pollution model and its application to the study, of $\mathrm{SO}_{2}$ distributions in the St. Louis metropolitan area, J. appl. Met. 13, 185-204.

Soeda T. and Omatu S. (1979) Real-time control of emissions in Japanese cities. Proc. of IIASA Workshop on Mathematical Models for Planning and Control of Air Quality (Edited by Fronza G. and Melli P.), Laxenburg, Austria.

Yanenko N. N. (1971) The Method of Fractional Steps, Springer-Verlag, Berlin.

Zannetti P., Melli P. and Runca E. (1977) Meteorological factors affecting $\mathrm{SO}_{2}$ pollution levels in Venice, Atmospheric Environment 11, 605-616. 


\section{RELATED IIASA PUBLICATIONS}

CP-81-25 Statistical Analysis of Winter Sulphur Dioxide Concentration Data in Vienna, by P. Bolzern, G. Fronza, and C. Überhuber.

CP-81-26 An Analysis of Finite Difference and Galerkin Techniques applied to the Simulation and Diffusion of Air Pollutants from a Line Source, by E. Runca, P. Melli, and F. Sardei.

WP-81-36 A practical numerical algorithm to compute steady-state ground level concentration by a $K$-model, by $E$. Runca (to be published in Atmospheric Environment).

Crop Yields in $\mathrm{CO}_{2}$-enriched Atmosphere, by P. Kauppi. IIASA Working Paper (forthcoming).

RR-78-9 The Smeared Concentration Approximation Method: A Simplified Air Pollution Dispersion Methodology for Regional Analysis, by R.L. Dennis.

RR-79-7 Assessment of Alternative Energy/Environment Futures for Austria 1977-2015, by W.K. Foell, R.L. Dennis, M.E. Hanson, L.A. Hervey, A.H. HoelzI, J.P. Peerenboom, and E. Poenitz.

RR-79-11 Real-Time Forecasting of Air Pollution Episodes in the Venetian Region. Part I: The Advection-Diffusion Model, by E. Runca, P. Melli, and A. Spirito. Part II: The Kalman Predictor, by G. Fronza, A. Spirito, and A. Tonielli.

Validation and Physical Parametrization of a Gaussian Climatological Model applied to a Complex Site, by E. Runca, A. Longhetto, and G. Bonino (to be published in Atmospheric Environment). 
